\title{
On the Stimulation and Maintenance of Chinese Learning Motivation of Preparatory Students
}

\author{
Jian Zhang \\ Nanchang Institute of Science \&Technology, Nanchang, 330108, China
}

Keywords: Chinese learning; motivation incentive; learning motivation

\begin{abstract}
Chinese language learning is the basis of the learning of other subjects. However, the characteristics of Chinese language lead to an awkward position of Chinese in all subjects. Generally speaking, the level of Chinese learning education is good, except in rural areas where the Chinese learning education is not optimistic, especially for some special college students-preparatory students. High language proficiency can only be achieved through long and continuous practice. Therefore, this paper explores how to stimulate and maintain the motivation of Chinese learning by focusing on the composition and influencing factors of Chinese learning motivation.
\end{abstract}

\section{Introduction}

Give a man a fish and you feed him for a day. Teach a man to fish and you feed him for a lifetime. In modern society, students are not required for rote memorization. What students are taught is not only knowledge, but also how to learn on their own. With the development of society, the national unity and harmony, more and more minority children go out of their hometown and integrate into the society, therefore, more and more ethnic prep students are accepted. Although their Chinese language learning level is low, the education sector generally believes that there are no bad students but only those who can not learn. So, more attention has been paid to how students learn and how to learn actively.

\section{Significance of Chinese Learning Motivation and Present Situation of Chinese Learning of Preparatory Students}

Learning motivation has a driving effect on learning, and it is like the effect of an engine on the operation of a car. The so-called motivation is to stimulate the individual to produce a particular behavior, so that the behaviour of the individual is directed towards a certain goal, lasting for a certain period of time. In general speaking, the generation and function of motivation is caused by internal displacement and external attraction. In general, the generation and function of motivation is caused by internal displacement and external attraction. Under the condition that the demand is not satisfied, the internal driving force is generated and when the internal demand of human being is imbalanced or absent, there will be needs. When an individual' demand is not met, it will stimulate the corresponding motivation which will motivate him to behave to meet his needs. Internal drive promotes individual's motivation and behavior, making the individual in the state of action. Motivation arises due to inducement, desire, and satisfaction. Teachers often encounter such learning problems in the course of teaching: students who have normal IQ but lack initiative to learn, students who are afraid of failure and unwilling to learn, and those who are too anxious to learn etc. The root cause of these problems lies in the motivation of learning. Teachers should have a thorough understanding of learning motivation, which is helpful to teaching effectively and helping students to learn. Learning motivation plays a great role in promoting students to learn with theoretical and practical guidance.

Chinese language is the common language of our country and the bearing of the whole national culture. Therefore, Chinese language is one of the most important basic tool subjects. Preparatory students live in places where their language proficiency is low since their early age. Even Chinese 
language is their second foreign language, and teachers use local language for their teaching. Apart from classes of learning Chinese language, they do not have too many opportunities and time to contact and use it. Therefore, there will be substandard pronunciation and these students are unable to express meaning freely in Chinese. It is even more difficult for students majoring in liberal arts to keep up with Classical Chinese learning and learning situation of students majoring in science is just so so. A poor level of Chinese language will seriously affect the future of university studies and social life. Under the background of intense educational competition, it is necessary to achieve good results in Chinese language teaching for preparatory students. We must explore to stimulate and cultivate students' Chinese learning motivation in the process of teaching.

\section{Influencing Factors of Chinese Learning Motivation}

There are two main types of internal and external influences:

Internal influencing factors, that is, the level of inducements determines the strength of attractiveness. A strong motivation will encourage people to accomplish their behavior actively as well as cultivate keen interest and strong desire for knowledge, leading to a strong motivation for learning and forming a very real internal motivation.

External factors:

Length of time. Time also has a certain impact on learning motivation. For example, the motivation of students who have just finished vacation is weaker than that of students who have studied for a short period of time in school, because with the extension of time, the influence of motivation on human behavior is weakened and can not be maintained.

Physical quality. Body is the basis for the conduct of activities.

Existence of other inducements. If we regard the importance of Chinese learning as an inducement, then in addition to this inducement, there are other inducements, such as the temptation to play, the temptation to go home, the temptation to sleep, and so on. The existence of other inducements will weaken the power of Chinese learning motivation.

Influence of environment. The atmosphere of Chinese language learning for preparatory students is bad, since most of them still take the local national language as the main communication language.

\section{Stimulation of Chinese Learning Motivation}

\subsection{Regular Emphasis on the Purpose of Learning}

This is an important method for students to produce learning needs. Through repeated emphasis, needs will emerge and then needs produce motivation. The purpose of learning can be to establish personal ideals or to set immediate learning goals. In the process of Chinese teaching, teachers make students understand the purpose and importance of Chinese learning, train students' ability of independent thinking and set up ideal. Chinese is the carrier of Chinese culture, and learning Chinese is also the inheritance and innovation of traditional culture. It is necessary to cultivate students' interest in Chinese characters and Chinese language. We are supposed to make learning tasks specified, such as weekly notes, reading a book per month, and so on. Data shows that students with specific goals are more likely to get good grades than those without clear goals. Because the vague and abstract goal gives students a kind of blank feeling, so that it is easy for students to lose their way and fail to find motivation. The combination of lofty ideal with the study goal can make students realize the short-term learning goal on the ground under the promotion of lofty ideal, and accumulate knowledge day by day, really promoting them to learn and grow. In carrying out study and education, we should try our best to avoid empty and boring preaching. From the perspective of students, we need to give full play to the interesting nature of the subject of Chinese and teach it imperceptibly, so that teachers can make use of the humanism of Chinese to let students know the cultural quality of the language. It is feasible to establish a reading club, from which students are capable of understanding the infinite richness and profoundness of Chinese 
language as well as form a desire to understand and learn the language. To learn Chinese well, students need more practice and then translate abstract knowledge and rules into practical application.

\subsection{Cultivation of Interest in Chinese Learning}

Motivation is not equal to interest. A good motivation to study a subject does not necessarily mean an interest in it. But interest in a subject deepens learning motivation, since interest is the best teacher. Interest produces a positive mental state of study. As for Chinese, knowledge which is closely related to life has great advantages in cultivating students' interest in Chinese learning.

\subsection{Appropriate Holding of Competition Activities}

The above measures are to help students generate learning needs, and thus generate internal drive. We know that extrinsic inducement is indispensable to motivation, so in teaching, teachers can use inducement to stimulate action. The study shows that in competitions, students have stronger motivation, more enthusiasm for learning and enhanced willpower to overcome learning difficulties if they have a desire to excel. Therefore, proper competition will improve learning efficiency, but too many competitions will lead to anxiety. In order to give full play to the motivation of competition, colleges and teachers can organize a variety of competitions in the classroom or on the campus, such as speech, composition competition, handwritten newspaper, etc.

\subsection{A Harmonious Relationship between Teachers and Students}

If teachers are welcome among students, it is very likely that subjects they teach are popular then students will actively learn this subject. Harmonious and friendly relationship between teachers and students is conducive to form a good learning environment. Therefore, teachers should not only respect students, treat them equally, communicate equally, learn together with them, but also should understand the characteristics of students because of their age, understand them, care for them, and finally, inspire them.

\section{Maintenance of Chinese Learning Motivation}

\subsection{Scientific Learning Methods}

In order to maintain students' learning motivation, teachers need to timely feedback students' learning situation and let them understand their own learning effects in a timely and effective manner, so as to enter the next learning stage. According to the Incentive Theory, when students know the results and their own improvement, they will increase their enthusiasm for learning, since there is a reward for giving. Besides, students can also find shortcomings, correct mistakes, inspire the urge for improvement, and strive for better. When teachers communicate with students, they should use praise and criticism properly to consolidate students' learning motivation. Teachers should analyze the psychological characteristics of students, and realize that different forms of feedback apply to different students. In the teaching feedback, teachers point out merits and demerits according to the actual situation. The comments should be enlightening, specific as well as easy for students to understand and students should not be evaluated simply by grade. Because of the fuzziness of Chinese subject, it is difficult for them to understand their learning situation in Chinese. Sometimes they do not show the fluctuation of learning condition in a short period of time, therefore, it is necessary for teachers to grasp the situation and communicate with students in time, making students know their own learning situation.

\subsection{Ensure the Correctness of Motivation}

Good motivation keeps students moving in the right direction. In order to improve students' learning, teachers should not only feedback their learning results, letting students know their own learning situation, but also help them analyze the learning results and summarize reasons. How students explain their learning outcomes will have an impact on their learning behavior. The correct attribution of success can make them realize the sense of achievement and increase their 
self-confidence. The correct attribution of failure will enable students to correct the mistakes made in the past, improve their study and obtain good results. Through scientific learning method-attribution guidance, it can help students to strengthen and maintain their motivation to learn Chinese, and reduce the phenomenon of giving up halfway.

\subsection{Make Use of Modern Scientific and Technological Means}

More and more colleges make use of multimedia classrooms. The development of multimedia promotes the development of modern educational technology. Different from the past, students can learn through video, music, pictures, etc. The teaching environment is optimized by multimedia means. Modern scientific and technological means can be used to organize more diverse learning activities, interesting and innovative learning activities, which not only enable students to learn knowledge in the process, but also to reduce psychological pressure on students. In addition, there will be more interaction between students and students as well as more interaction between students and teachers during activities. This promotes feelings between students and teachers, making it more effective in maintaining motivation.

\subsection{High Quality Teachers}

For students, teacher has played the role of guide and tutor. In order to stimulate and maintain students' learning motivation for Chinese subjects, the requirements for teachers are more severe. They are asked to fully master the subject knowledge, deal well with students, learn and use incentive mechanisms and attribution guidance, stimulate, cultivate and maintain students' good motivation for Chinese learning, timely help them when they have difficulties and puzzles, letting them master scientific learning methods and learn to study independently, give full play to the role of teachers, set a good example, so that students can experience the fun of learning, establish self-cognition, confidence, self-thinking and so on. In the process of teaching, teachers should adopt appropriate ways to train students according to relevant scientific theories such as Reinforcement Theory.

\section{Conclusion}

Learning motivation stimulates students to carry out learning activities by triggering behavior. By some methods, students can produce their own learning motivation, but teachers can make the level of learning motivation stimulation higher and offer guidance for students, thereby maintaining their learning motivation. Teachers can take appropriate measures to stimulate and maintain the motivation of Chinese learning, because preparatory students are not officially admitted as freshmen. Compared with the formal ones, preparatory need to try their studies in universities for a period of time. Moreover, preparatory students are from ethnic minorities and are affected by their own traditional culture, so they do not have a good master of Chinese, which demands learning motivation more. In order to help them, teachers are required to understand and help them by improving their teaching objectives. Motivation plays a very important role in learning, so it is very useful to stimulate and maintain learning motivation, especially for Chinese learning motivation. Chinese is involved in all aspects of life, so learning Chinese well can help us to communicate better. To stimulate and maintain students' learning motivation is the main point of teaching.

\section{References}

[1] Chen Hongxiu. On the Motivation and Maintenance of Chinese Learning Motivation [J]. Journal of Jiangsu Institute of Education (Social Science Edition), 2002(06):105-106.

[2] Wang Qinhai. On the Stimulation and Maintenance of College Chinese Learning Motivation [J]. Journal of Changsha Aeronautical Vocational and Technical College, 2004(01):7-8+11.

[3] Luo Shuanglan, Li Mang. A Review of Relevant Researches on How to Stimulate Learning Motivation [J]. Journal of Guangxi Normal University (Natural Science Edition), 2004(04):95-100.

[4] Zhang Liyuan. On the Cultivation and Stimulation of Learning Motivation in Chinese Teaching [J]. Intelligence, 2011(22):112. 